\title{
Nucleation in Ising ferromagnet by a field spatially spreading in time
}

\author{
Muktish Acharyya \\ Department of Physics, Presidency University \\ 86/1 College Street, Calcutta-700073, India \\ E-mail:muktish.physics@presiuniv.ac.in
}

\begin{abstract}
The nucleation in Ising ferromagnet has been studied by Monte Carlo simulation. Here, unlike the earlier studies, the magnetic field is spreading over the space in time. The nucleation time is observed to increase as compared to that in the case of static field. The clusters of negative spins is observed to grow from the center. The growth of effective magnetisation is studied with temperature and the strength of spreading magnetic field. The ratio of nucleation time and effective time is also studied with strength of spreading magnetic field. The effective time would introduce itself as a new scale of time in the case of nucleation by spatially spreading magnetic field.
\end{abstract}

PACS Nos.: $05.50+\mathrm{q}$

Keywords: Ising model, Monte Carlo simulation, Nucleation 


\section{Introduction:}

The dynamical aspect of Ising ferromagnet, particularly concerned with the nucleation and growth of clusters, has become an active area of theoretical research over a very long period of time[1,2]. The lifetime of metastable states and its dependences on the temperature and applied magnetic field are main focus of attention. Extensive Monte Carlo studies are performed in last few decades[3, 4, 5]. The large scale simulational results[3] show strong agreement with classical nucleation theory. Some of the important studies may be mentioned briefly as follows: classical nucleation theory was reexamined by extensive Monte Carlo simulations and connection with the hysteresis was found [3]. In this case, the applied magnetic field was taken uniform over the space and constant in time. Later, the nucleation in Ising ferromagnet was studied in presence of a time dependent magnetic field to analyse the hysteresis phenomenon. However, the field was uniformly distributed over the space[2]. The magnetisation reversal was studied in Ising ferromagnet by a pulsed field[6]. The magnetisation reversal was also studied in Ising ferromagnet by a periodic impulsive field [7]. Magnetisation switching behaviour was also studied [5] in vector spin model with long range dipolar interaction by Monte Carlo simulation. The magnetisation reversal and switching were studied experimentally $[8,9]$. It may be noted here all theoretical and experimental investigations are made in the presence of either a static magnetic field or by a field varying in time[4]. In this context, it should be mentioned that, the recent Monte Carlo study[10] yields a heat assisted magnetisation reversal by a decaying and spatially spreading temperature pulse. This study is technologically important for high density ultrafast magnetic type recording. But in the earlier studies on the magnetisation reversal by time dependent magnetic field, the field was distributed uniformly over the space. No such study was found where the spatio-temporal variations of field was considered, in the case of nucleation in Ising ferromagnet. It is interesting to know how the magnetsation reversal happens when the field is spreading spatially in time.

In the present paper, the nucleation in the Ising ferromagnet was studied where the field is spreading spatially in time. The Monte Carlo simulation with Metropolis single spin flip dynamics [11] is used here. In section-II the model and the simulation scheme are described. The simulational results are reported in section-III. The paper ends with summary in section-IV.

\section{The model and simulations:}

The Hamiltonian representing the Ising feromagnet (described on a square lattice of linear size $L$ ) in the presence of a magnetic field is

$$
H=-J \sum_{<i j>} S_{i}^{z} S_{j}^{z}-\sum_{i} h(r, t) S_{i}^{z} .
$$


Where, $S_{i}^{z}(= \pm 1)$ is the Ising spins, $J$ is the ferromagnetic $(J>0)$ interaction strength and $h(r, t)$ is the space and time dependent magnetic field. The spatially spreading magnetic field is taken as

$$
h(r, t)=-h_{0} \exp \left(-\frac{\left(\left(x-x_{0}\right)^{2}+\left(y-y_{0}\right)^{2}\right)}{2\left(a^{2}+b^{2} t^{2}\right)}\right) .
$$

The field $h(r, t)$ is acting along -z direction and spreading in time $(t)$ radially $\left(r^{2}=\left(x-x_{0}\right)^{2}+\left(y-y_{0}\right)^{2}\right)$ outward from the central site $\left(x_{0}, y_{0}\right)$. This is like a unnormalised Gaussian, spreading radially keeping its height $\left(h_{0}\right)$ fixed. The factor $h_{0}$ is defined as the strength of the field. Hence, $h_{0}$ is the magnitude of the spreading field (measured in the unit of $J$ ) at the central site for ever. The value of the field decreases from that at the central site $\left(x_{0}, y_{0}\right)$ as one go radially away from the origin. The effective radius $\left(r_{\text {eff }}\right)$ is defined as that value of the radius where the value of the field becomes $\frac{1}{e}$ times the value at origin. So, $r_{\text {eff }}=\sqrt{\left(2\left(a^{2}+b^{2} t^{2}\right)\right.}$. At time $t=0$ the effective radius was $\sqrt{\left(2 a^{2}\right)}$. As time passes the effective radius $r_{\text {eff }}$ increases and eventually the field spreads over the whole lattice. The nearest neighbour ferromagnetic uniform $(J=1)$ interaction is considered here. The boundary condition is periodic in both sides of the square lattice.

A square of linear size $L=(101)$ is considered and the origin of spreading field $h(r, t)$ is taken at the center $\left(x_{0}=51, y_{0}=51\right)$ of the lattice. Initially (at $t=0$ ) value of all spins $\left(S_{i}^{z}\right)$ are taken up $(+1)$. In the random spin updating scheme. a site is chosen randomly and the energy of the system is calculated (by using equation (1)). The probability of flip of this chosen spin is obtained from Metropolis rate[11]

$$
P\left(S_{i}^{z} \rightarrow-S_{i}^{z}\right)=\operatorname{Min}\left[\exp \left(-\frac{\Delta H}{k_{B} T}\right), 1\right]
$$

where, $k_{B}$ is Boltzmann constant and $T$ denotes the temperature (measured in the unit of $J\left(k_{B}\right) . \Delta H$ is the change in energy due to spin flip. Using Monte Carlo method, this spin flipped $\left(S_{i}^{z} \rightarrow-S_{i}^{z}\right)$ if the value of Metropolis rate is less than or equal to a fraction $(0<f<1$ and distributed uniformly) chosen randomly. $L^{2}$ such random updates of spins constitutes one Monte Carlo Step per Spin (MCSS) and acts as the unit of time in the simulation. Here, $h(r, t)$ is updated after each MCSS.

\section{Results:}

The Monte Carlo simulation is performed on a two dimensional lattice of linear size $L$. Here $L=101$, so that the site $(51,51)$ becomes the central site. The parameters $a$ and $b$ of the the spreading field were kept constant ( $a=1.0$ and $b=0.1$ ) throughout the simulational study. Later, the field was 
allowed to spread according to the equation (2) and the spins were updated (randomly) with probability (given in equation -3 ). The total magnetisation $m(t)$ is calculated as $m(t)=\frac{\sum S_{i}^{z}}{L^{2}}$ and if its value becomes less than or equal to 90 percent of its initial value, it nucleates and the time required for this is called the nucleation time $\tau_{\text {nucl }}$. This prescription of calculating the nucleation time was used earlier [3].

It was observed that a single compact cluster of down spins, embedded in the sea up spins, grows from central region. A typical growth of a cluster of down spins is shown in figure- 1 . Here, $T=0.8$ and $h_{0}=1.5$ are chosen. This resuls is quite obvious here, since the field is spreading radially. As time passes the boundary sites of negative spin cluster experiences larger (than that at earlier time) values of negative field and get flipped. In this way the cluster of down spins grows. From figure-1(a) and figure-1(b), it is also observed that, in earlier time, the topology of the lattice is not reflected in the growing clusters of negative spins. However, for later time, as the field spreads radially, this topological symmetry of the lattice starts to appear from the shape of the growing cluster. It may be noted that figure-1(c) and figure-1(d) show the clusters of nearly square shaped.

The growth and the nucleation studied here (in the presence of a magnetic field spatially spreading in time) is compared quantitatively with that in the presence of a uniform and static magnetic field. The nucleation time was calculated as averaged over 20 different random samples. In this case one expects that for higher values of field strength the nucleation time (for spreading field) would be many times higher than that for uniform and static field. The reason is very clear here. In this case all spins will not experience the maximum amount of field at any given instant (MCSS). However, this time (for spreading field) would become of the same order of that for uniform and static field as one apply the low field. Here, the nucleation time is quite large for the case of static field. And for spreading field, the field will get sufficient time to spread over the whole lattice. As a result, the nucleation time $\left(\tau_{\text {nucl }}\right)$ for spreading field will be of the same order of that of static field. The total number of downspins, $N_{\text {eff }}^{\downarrow}$, within the circle of effective radius $\left(r_{\text {eff }}\right)$ is also calculated. Hence, the magnitude of effective magnetisation, $\left(m_{\text {eff }}\left(=\frac{N_{\text {eff }}^{\downarrow}}{N_{\text {total }}}\right)\right.$, is calculated. $N_{\text {total }}$ is equal to the total number of spins, i.e., $L^{2}$. As time passes, the $m_{\text {eff }}$ increases. A new time scale, called effective time $\left(\tau_{e f f}\right)$, may be defined here as follows: the time required by the magnitude of the effective magnetisation to reach the value 0.01 . This effective time is a time associated to the developement of local magnetisation within the circle of radius $r_{\text {eff }}$.

These results are depicted in figure-2. This study clearly indicates the difference in time of the growth and nucleation for different types of fields (uniform-static type and spreading type). 
The growth of effective magnetisation $\left(m_{\mathrm{eff}}\right)$ at a fixed temperature $(T=$ $0.80)$ and for the different values of the strength of the magnetic field $\left(h_{0}=\right.$ $0.60,0.65,0.80)$ are studied. These are shown in figure-3. For any fixed value of field strength, the growth $m_{\text {eff }}$ is very slow and suddenly it starts to grow very rapidly. Naturally, the $\tau_{\text {eff }}$ will increase for smaller values of the strength of spreading magnetic field at any given temperature of the system. This feature is clear from figure- 3 .

The growth of $m_{\text {eff }}$ is also studied for a fixed value of field strength $\left(h_{0}=\right.$ $0.8)$ and different values of temperature $(T=0.8,1.0$ and 1.2). This is shown in figure-4. Here, also it is observed that the effective time $\left(\tau_{\text {eff }}\right)$ increases as the temperature decreases, for a fixed value of the maximum magnitude of the spreading magnetic field. It may be mentioned here, that in the case of nucleation by static field, at $T=0.8, h_{0}=1.5$ would be in the multidroplet region and $h_{0}=0.8$ would be near the crossover of multidroplet and singledroplet region for the system size considered here [1].

To consider the effective time $\left(\tau_{\text {eff }}\right)$ as a new time scale, in the case of nucleation in Ising ferromagnet, would be justified in the following study. This is the main part of this paper. The ratio $\left(R=\frac{\tau_{\text {nucl }}}{\tau_{\text {eff }}}\right)$ of nucleation time $\left(\tau_{\text {nucl }}\right)$ to the effective time $\left(\tau_{\text {eff }}\right)$ is studied as a function of the strength of spreading magnetic field for different values of temperature. This is shown in figure-5. The ratio $(R)$ shows a nonmonotonic variation with the strength of spreading magnetic field. The reason behind this nonmonotonic variation of $R$ with $h_{0}$, is due to the growth of the clusters formed by the negetive spins, which happens only within the circle of effective radius ,i.e., $r_{\text {eff }}$. The lifetime of metastable state, i.e., $\tau_{\text {nucl }}$, is solely dependent on the time of growth of negative clusters of a required value of magnetisation. However, in the present study, this is solely governed by a single compact cluster of negative spins which resides within the circle of effective radius $\left(r_{\text {eff }}\right)$. The rate of spreading $\left(\frac{d h(t)}{d t}\right)$ of the field, is a function of time and the radius $\left(r_{\text {eff }}\right)$ of the effective circle. So, it is expected that the nucleation time $\left(\tau_{\text {nucl }}\right)$ and the effective time $\left(\tau_{\text {eff }}\right)$ have different types of dependence on $h_{0}$. Hence, $R$ would show a nonmonotonic (nonlinear) dependence on $h_{0}$.

As the field increases, the ratio increases first, and then reaches a maximum, then it starts to decrease. Since the rate of spreading of the field, is not constant, there may exist some value of the field, for which the nucleation time $\left(\tau_{\text {nucl }}\right)$, differs widely, from effective time $\left(\tau_{\text {eff }}\right)$. Here, in this region near the peak, the growth of $m_{\text {eff }}$ becomes much slower than the growh of $r_{\text {eff }}$. Hence, $\tau_{\text {nucl }}$ becomes much larger than $\tau_{\text {eff }}$ and consequently $R$ becomes maximum. This is a possible qualitative explanation of getting a peak of $R$ for a definite value of $h_{0}$. However, further extensive simulational investigation is required to have some quantitative idea about the specific value of $h_{0}$ which maximises $R$. Precisely, the $R$ takes the value, depending on $h_{0}$, appears to be the competition of rates of growth of $m_{\text {eff }}$ and the magneti- 
sation for reversal. The maximum of $R$, is that value, where the nuclation time is relatively high for a particular value of $h_{0}$. This has an experimental significance also. The reversal or switching time may be obtained accordingly if the amplitude of the spreading field is suitably adjusted. This may be used in magnetic storage device to have an optimum condition of speed of recording and the longivity of the recorded media.

This indicates that the effective time $\left(\tau_{\text {eff }}\right)$ may be considered as to define a new scale of time in the problem of nucleation in Ising ferromagnet in the presence of a spreading magnetic field. If the effective time would not provide another scale of time, this would be a simple factor of nucleation time. Consequently, if the ratio of nucleation time and the effective time was plotted against the applied field strength, it would show a straight line parallel to the horizontal axis. However, in this case, $R$ shows a nonmonotonic variation with $h_{0}$. The maximum value of the ratio $\left(R_{\max }\right.$ and the strength of the field $\left(h_{0}^{\max }\right.$ ) for which $R$ becomes maximum, is plotted against the temperature $(T)$ and shown in figure-6. Both $h_{0}^{\max }$ and $R_{\max }$ decreases nonlinearly as the temperature increases.

\section{Summary:}

In this paper, the nucleation in Ising ferromagnet is studied by Monte Carlo simulation using Metropolis single spin flip dynamics. All earlier studies are performed with magnetic field uniformly distributed over the space and constant in time. A few studies are done by using a slowly varying (in time) magnetic field but uniform over the space. However, the present study differs significantly from all other earlier studies. Here, the applied magnetic field has a spatio-temporal variation. The applied magnetic field is spreading over the space in time. A Gaussian-like simple form of spreading magnetic field is taken here. The width of the Gaussian is spreading in time keeping the altitute fixed.

The nucleation time would become larger (for fixed values of temperature and field strength) in comparison to that observed in the case of static and uniform magnetic field. In the case of static and uniform magnetic field, the nucleation time provides the scale of time. A considerable number of studies are done using this time scale which was also considered as the lifetime of the metastable state. However, in the case of spreading magnetic field, apart from the nucleation time, a new scale of time appears. This is the effective time. Interestingly, it is observed in the present study, the variation of this ratio with field strength is nonmonotonic. Hence, one would say that the effective time and nucleation time provide separately different scales of time in this problem of nucleation in spreading magnetic field.

The effective time and the nucleation time are studied here as a function of the factor predominantly determining the rate of spreading of the field. It 
is observed that both show similar power law type decay.

The study of the nucleation in the presence of a spreading magnetic field would help to sustain the metastability for a longer period if one uses the magnetic field is spreading (in time) very slowly. In the field of technology of magnetic recording, this may help to increase the longivity of recorded media, if the rate of spreading is suitably managed. Hopefully, this study will also explore some new class of problems in nonequilibrium statistical mechanics in near future.

Acknowledgments: The library facility provided by the Central Library of Calcutta University is gratefully acknowledged. Author would like to thank D. Stauffer for important discussions. 


\section{References:}

1. P. A. Rikvold and B. M. Gorman, Annual Reviews of Computational Physics I, edited by D. Stauffer (World Scientic, Singapore, 1994), pp. 149-191;

2. S. Wonczak, R. Strey, D. Stauffer, J. Chem. Phys., 113, 1976 (2000); K. Park, P. A. Rikvold, G. M. Buendia, M. A. Novotny, Phys. Rev. E, 49, 5080 (1994); H. Vehkamaki and I. J. Ford, Phys. Rev. E, 59 6483 (1999); K. Brendel, G. T. Barkema and H. van Beijeren, Phys. Rev. E 67, 026119 (2003); K. Brendel, G. T. Barkema and H. van Beijeren, Phys. Rev. E 71, 031601 (2005); G. M. Buendia, P. A. Rikvold, Kyungwha Park, and M. A. Novotny, J. Chem. Phys., 121, 4193 (2004); S. Ryu and W. Cai, Phys. Rev. E, 81, 030601(R) (2010); H. Chen and Z. Hou, Phys. Rev. E, 83, 046124 (2011); C. Bosia, M. Caselle, D. Cora, Phys Rev E 81021907 (2010).

3. M. Acharyya and D. Stauffer, European Physical Journal B, 5571 (1998).

4. P. A. Rikvold, H. Tomita, S. Miyashita and S. W. Sides, Phys. Rev. Lett., 92, 015701 (2003).

5. D. Hinzke and U. Nowak, J. Magn. Magn. Mater., 221365 (2000) 52, $311(2000)$.

6. A. Misra and B. K. Chakrabarti, Europhys. Lett.

7. M. Acharyya, Physica Scripta 82065703 (2010).

8. D. Hinzke, N. Kazantseva, U. Nowak, R. Chantrell, Phys. Rev. B, 81, 174428 (2010).

9. K. Vahaplar, A. M. Kalashnikova, A. V. Kimel, S. Gerlach, D. Hinzke, U. Nowak, R. Chantrell, A. Tsukamoto, A. Itoh, A. Kirilyuk, Th. Rasing, Phys. Rev. B, 85, 104402 (2012).

10. W. R. Deskins, G. Brown, S. H. Thompson, P. A. Rikvold, Phys. Rev. $B, \mathbf{8 4}, 094431$ (2011).

11. K. Binder and D. W. Heermann, Monte Carlo simulation in statistical physics, Springer Series in Solid State Sciences, (Springer, -York, 1997). 

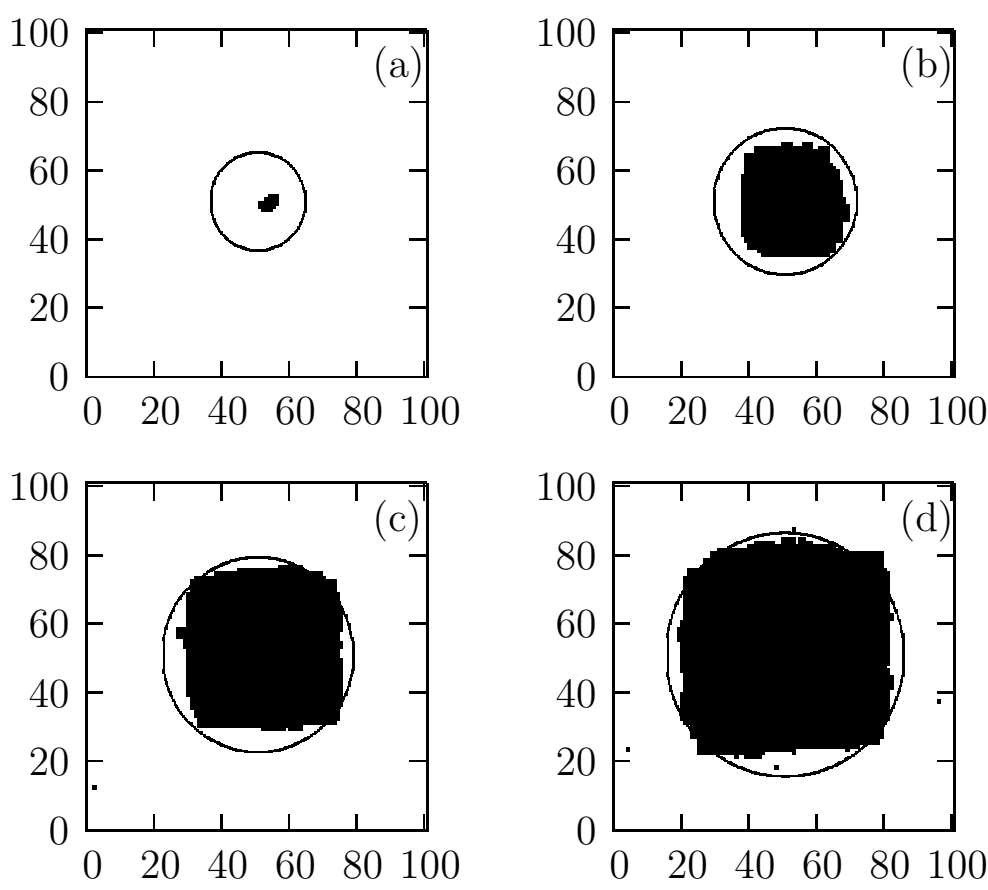

Fig.1 Growth of negative spin clusters (marked by black dots) (on a 101×101 square lattice) with time $(t)$. The solid line is a circle of radius $r_{\text {eff }}$ in each diagram. Here, $T=0.8$ and $h_{0}=1.5$. (a) $t=100 \mathrm{MCSS}$, (b) $t=150 \mathrm{MCSS}$, (c) $t=200$ MCSS and (d) $t=250$ MCSS. Here, $a=1.0$ and $b=0.1$. 


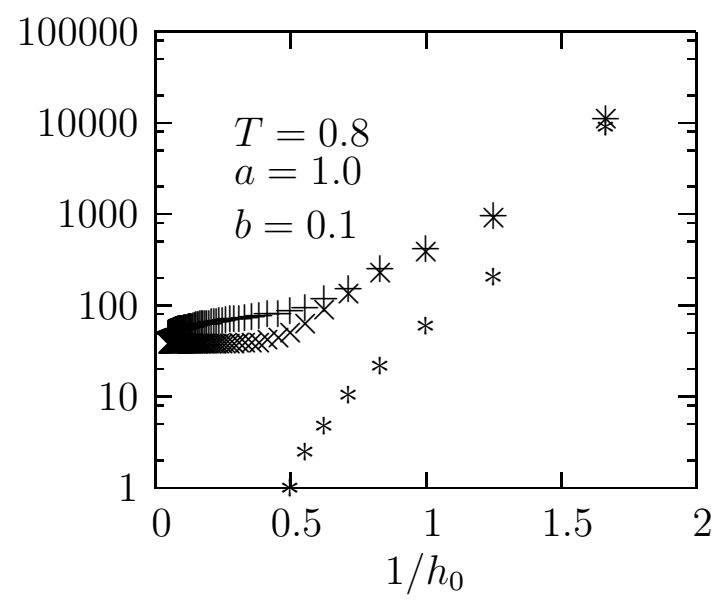

Fig-2. Dependences of $\tau_{\text {nucl }}(+), \tau_{\text {eff }}(\times)$ with the amplitude $\left(h_{0}\right)$ of spreading field and that of $\tau_{\text {nucl }}(*)$ with the magnitude $\left(h_{0}\right)$ of static uniform field. Here, $T=0.8, a=1.0$ and $b=0.1$. 


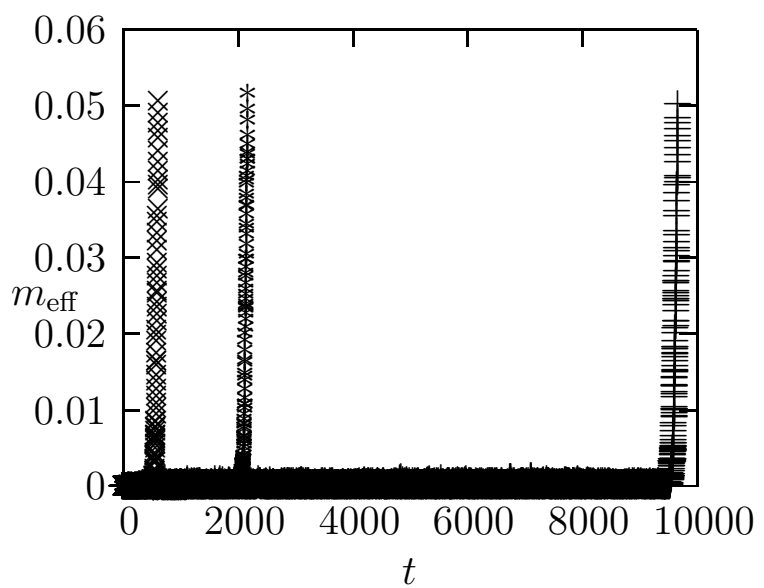

Fig-3. The effective magnetisation $\left(m_{\mathrm{eff}}\right)$ is plotted against the time $(t)$. Different symbols corresponds to different values of amplitudes $\left(h_{0}\right)$ of the spreading field. $h_{0}=0.60(+), h_{0}=0.65(*)$ and $h_{0}=0.80(\times)$. Here, in all cases $T=0.8, a=1.0$ and $b=0.1$. 


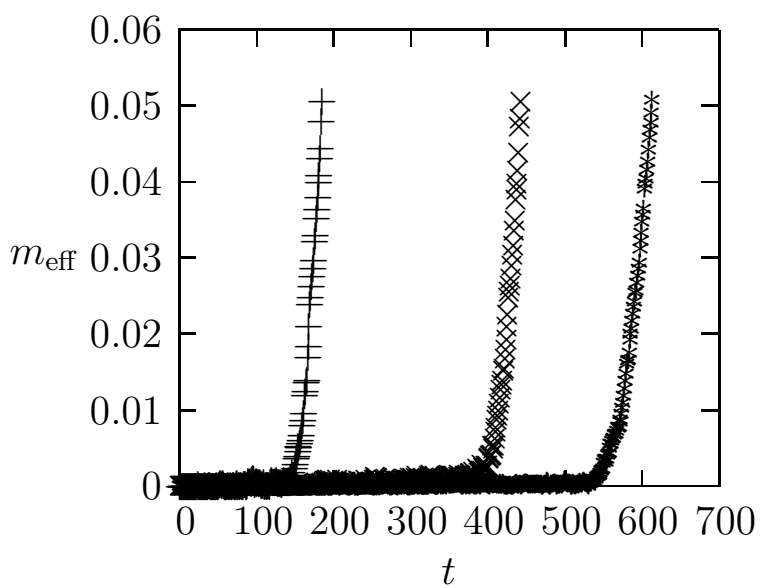

Fig-4. The effective magnetisation $\left(m_{\text {eff }}\right)$ plotted against time $(t)$. Different symbols corresponds to different values of temperatures $(T) . T=1.2(+)$, $T=1.0(\times)$ and $T=0.8(*)$. Here, the value of field strength $h_{0}=0.8$, $a=1.0$ and $b=0.1$. 


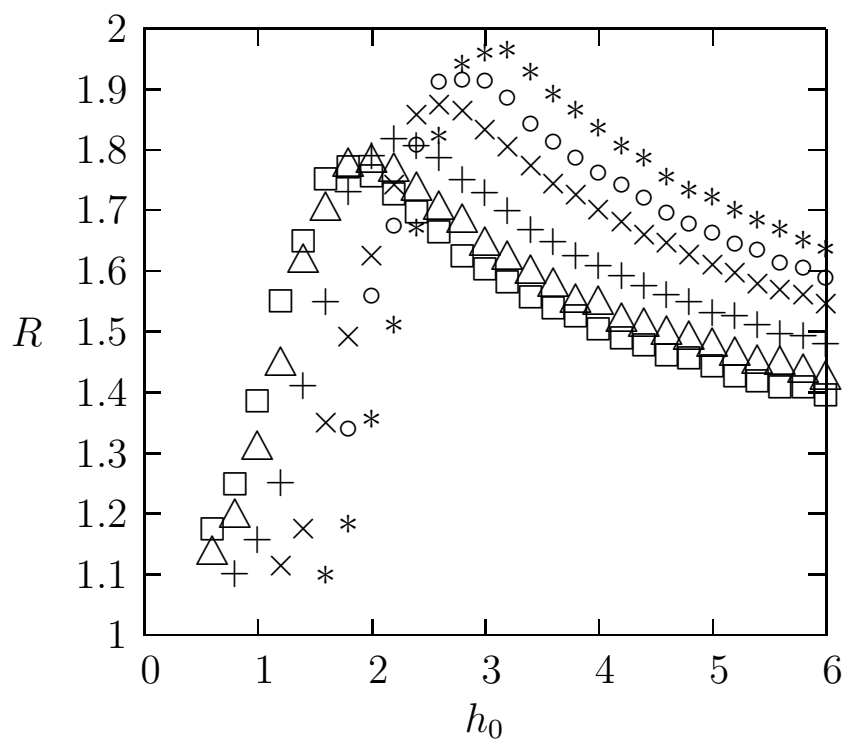

Fig-5. The ratio $\left(R=\frac{\tau_{\text {nucl }}}{\tau_{\text {eff }}}\right)$ is plotted against the field strength $\left(h_{0}\right)$ for different temperatures $(T)$. Different symbols corresponds to different temperatures. $T=1.3(\square), T=1.2(\triangle), T=1.0(+), T=0.8(\times), T=0.7(\circ)$ and $T=0.6(*)$. Here, $a=1.0$ and $b=0.1$. 


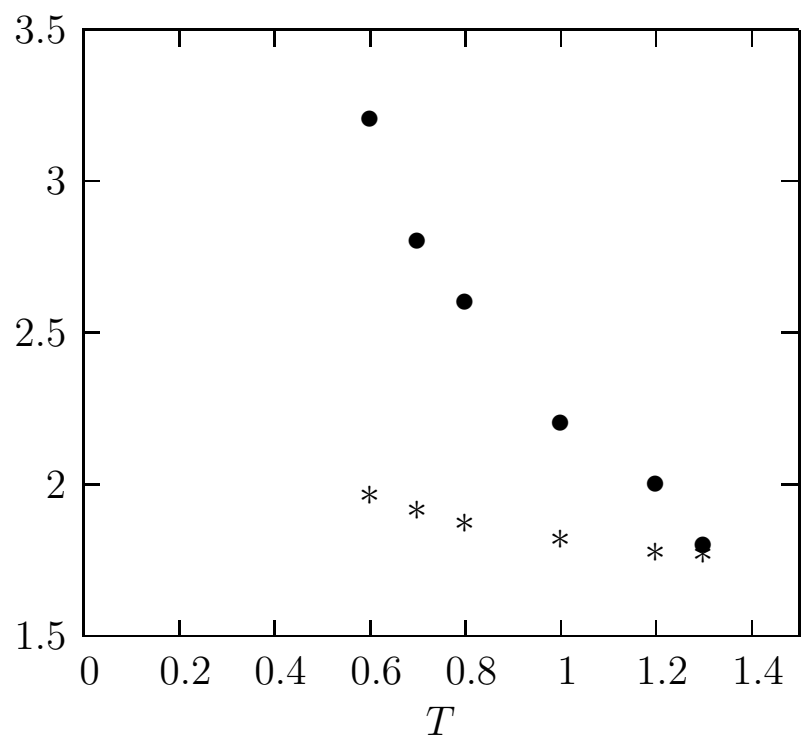

Fig-6. The $h_{0}^{\max }(\bullet)$ and $R_{\max }(*)$ plotted against the temperature $(T)$. Here, $a=1.0$ and $b=0.1$. 